\title{
Assessment of the Evaluation Framework for Energy Maximising Control Systems for the Wavestar Wave Energy Converter
}

\author{
Christian Windt $^{1,2}$, Nicolas Faedo ${ }^{1}$, Markel Penalba ${ }^{1}$ and John V. Ringwood ${ }^{1}$
}

\begin{abstract}
During the design process and evaluation of energy maximising control systems (EMCSs) for wave energy converters (WECs), control techniques rely heavily on numerical modelling. For fast computation, these numerical models are mostly based on low-fidelity boundary element method (BEM) codes and linear hydrodynamic models. However, to ensure optimal performance in a physical environment, more realistic, high-fidelity numerical frameworks, such as Computational Fluid Dynamics (CFD) based numerical wave tanks (CNWTs), should be considered during the evaluation of EMCSs. This paper investigates the influence of different numerical evaluation frameworks on the performance evaluation of EMCSs. The Wavestar WEC, subject to three different EMCSs with varying aggressiveness, i.e. resistive, reactive and moment-based control, is chosen as the case study. Results show that more aggressive EMCSs require high-fidelity numerical modelling to correctly evaluate their performance.
\end{abstract}

\section{INTRODUCTION}

To moderate global warming and reduce the emission of greenhouse gases, renewable energies are vital to satisfy the increasing global energy demand. Amongst others, WECs have a strong potential to contribute to the energy supply [1]. To be commercially viable, it is well known that devices

(a) have to be installed in arrays of multiple WECs [2]

(b) should be equipped with EMCSs [3].

The development of EMCSs can be split into two consecutive parts: design and evaluation. During the design the fundamental concept and the mathematical model are conceived. During the evaluation process the performance of the controller is assessed. For both steps researchers rely heavily on numerical modelling.

In classical control applications, the mathematical models, used for the design stage, are often linearised around a desired operational point, according to the process under analysis. The controller is subsequently synthesised to drive the system towards this point and, thus, in the neighborhood of this operational point, the linearising assumption for the controller evaluation is also a valid approach, delivering trustworthy results. During the design of controllers for wave energy converters, linear hydrodynamic models are also widely applied. For example, linear BEM based models are well established, delivering results at acceptable computational cost. These models assume small amplitude device motion but lose fidelity under large WEC motion. The objective of WEC device control is to drive the system towards resonance with the incoming wave field and, thus,

\footnotetext{
${ }^{1}$ Centre for Ocean Energy Research, Maynooth University, Co. Kildare, Ireland

${ }^{2}$ christian.windt.2017@mumail.ie
}

increase the device motion and power production. By way of example, Fig. 1 shows the operational space for a generic WEC without and with (latching) control applied [4]. A clear increase of the operational space can be observed when considering a controlled WEC. The large amplitude motions may result in viscous drag, flow separation, vortex shedding and other non-linear hydrodynamic effects. Contrary to the aforementioned classical control applications, the energymaximising operating conditions do not comply with the linear assumption in the hydrodynamic model.

Linear BEM-based numerical wave tanks (BNWTs) have been extended to included non-linear Froude-Krylov forces [5] and viscous (drag) effects [6]. While the former can be determined, for some devices, through analytical solutions, viscous drag effects are usually based on empirical models for the drag coefficient. Inherently considering viscous drag and turbulence effects, CFD-based numerical wave tanks (CNWTs) deliver higher hydrodynamic fidelity but come at significantly increased computational cost, limiting their application to specific case studies.

Recently, a number of studies have begun to incorporate EMCSs into CNWT experiments to allow more realistic performance evaluation. Giorgi et al. [4] describe the implementation of latching control in a CNWT, which is used to evaluate the optimal latching duration for a heaving point absorber. Davidson et al. [7] implement proportional-integral control, where the parameters are selected using system identification techniques. Comparing the CNWT results against BNWT experiments shows an overestimation of the WEC motion and power absorption in the BNWT. In [8], an adaptive EMCS, based on a receding-horizon pseudospectral optimal control formulation, is implemented and evaluated in a CNWT. Dolguntseva et al. [9] include stroke length control within the mathematical power take-off (PTO) model by the means of end stop spring forces. In [10], passive control is realised by replicating a pressurised pneumatic

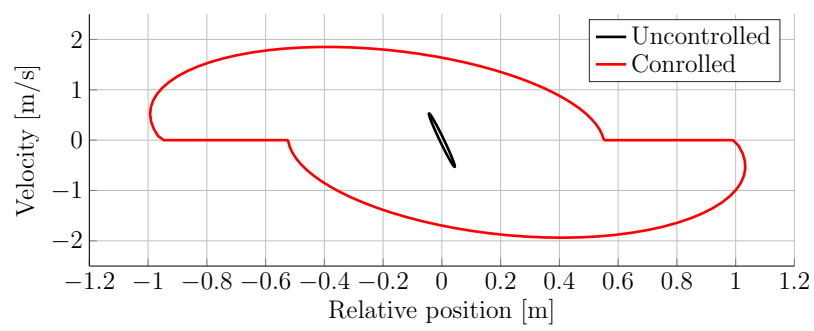

Fig. 1: Operational space for a WEC with and without control applied (adapted from [4]) 
cylinder, i.e. air spring, in the numerical model providing a negative spring force on the PTO system. Penalba et al. [11] implement passive and reactive controllers in a verification and evaluation study of a high-fidelity wave-towire simulation platform. Most recently, Windt et al. [12] highlight the challenges arising from the implementation of EMCSs in CNWTs, based on the case study of a reactively controlled moored point-absorber type WEC.

Although some implementations of EMCSs in CNWTs can be found in the literature, for the design of EMCSs, these models are still computationally too costly and are infeasible for real-time control. Thus, development of EMCSs relies mainly on BNWTs. This poses the concern that EMCSs are optimised in a hydrodynamically low-fidelity modelling framework and indicate optimal power output when evaluated in BNWTs, but do not necessarily perform well when evaluated in a more realistic, high-fidelity framework, such as a CNWT and, ultimately, in the physical world. It has been reported in [13] that the majority of the EMCS are not evaluated in realistic evaluation frameworks. In fact, they are usually tested using similar (linear) models as the ones used during the design process and thus distort the results obtained from the evaluation.

In this paper, a CNWT and linear BNWT model of the Wavestar device are employed to investigate the influence of different numerical evaluation frameworks on the performance evaluation of EMCSs for WEC devices. The performance of the EMCSs will be evaluated by comparing the device dynamics of the Wavestar WEC subject to three different EMCSs:

1) moment-based energy-maximising control [14]

2) reactive output feedback (displacement and velocity) control with a spring-damper

3) resistive output feedback (velocity) control with a damper.

To different extends, the three EMCSs will drive the WEC away from the linear assumption in hydrodynamic model, dependent on the aggressiveness of the controller, with the resistive controller being the least aggressive and the moment-based control the most aggressive.

The remainder of the paper is organised as follows. Section II presents the case study considered herein. Section III details the numerical wave tank setups. Following, Section IV describes the employed EMCSs. Section V then presents and discusses the results. Finally, conclusions are drawn in Section VI.

\section{CASE STUDY}

The Wavestar WEC (see Fig. 2) is considered as case study. The device consists of a hemispherical hull with an operational degree of freedom (DoF) in heave. The hydraulic power take-off (PTO) system consists of a hydraulic cylinder, pumping hydraulic fluid through a hydraulic motor. The device depicted in Fig. 2 was deployed at Hanstholm, Denmark. The rated power of the full scale device with 20 floats is $500[\mathrm{~kW}][15]$.
In the experimental tank test campaign documented in [16], a $1 / 5^{\text {th }}$ scale device has been tested. The physical prototype in the ocean basin at Plymouth University and a schematic of this device, including the main dimensions, are depicted in Figs. 4 and 3, respectively. Validation of the CNWT model used in this study is based upon the $1 / 5^{\text {th }}$ scale tests and is documented in [17].

In this study, a single, irregular JONSWAP sea state with a characteristic wave height $H s=0.15[\mathrm{~m}]$ and period $T p=1.4[\mathrm{~s}]$ is tested. These characteristics are based upon the experimental tank tests documented in [16]. The simulation duration is $t_{\mathrm{sim}}=140[\mathrm{~s}](=100 T p)$. Plots of the free surface elevation (FSE) and spectral density distribution (SDF), measured at the device location during preliminary empty tank simulations, are shown in Figure 5. PTO cylinder displacement, velocity and force are extracted from the simulations and used for the comparison of the evaluation frameworks.

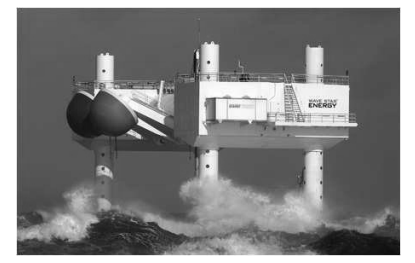

Fig. 2: Full scale Wavestar device deployed at Hanstholm, Denmark (adapted from [15])

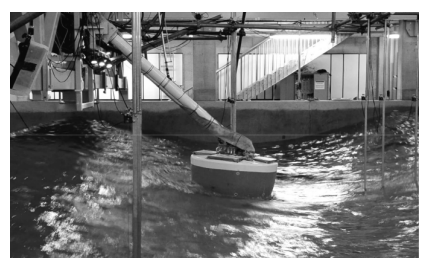

Fig. 3: 1:5 scale Wavestar device in the ocean basin at Plymouth University (adapted from [18])

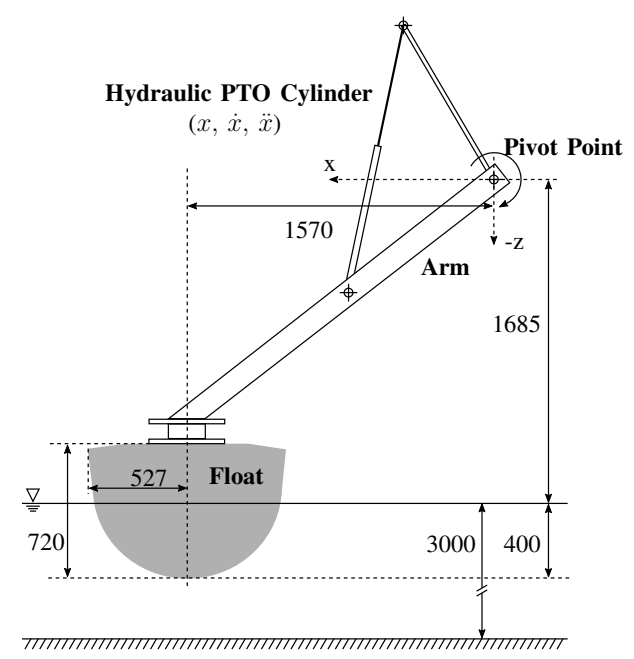

Fig. 4: Schematic of the $1 / 5^{\text {th }}$ scale Wavestar device: all dimensions in [mm] (adapted from [17])

\section{NUMERICAL WAVE TANKS}

\section{A. Linear BNWT}

In linear time-domain hydrodynamic BNWT models, the dynamics of the device respond to the classic linear hydrodynamic formulation [19], based upon Cummins equation [20] 
(a) Free surface elevation

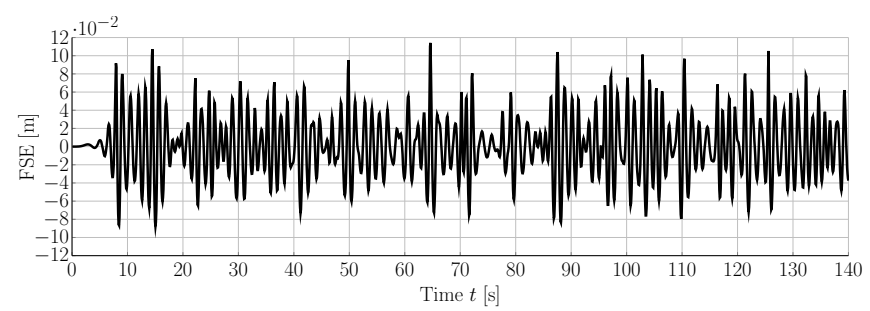

(b) Spectral density distribution

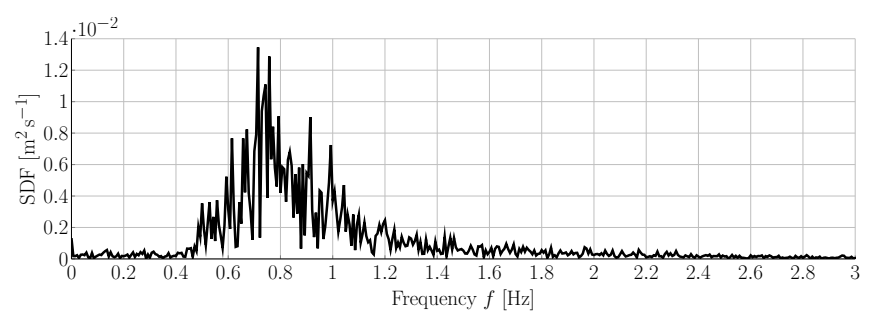

Fig. 5: FSE and SDF of the irregular JONSWAP sea state with $H s=0.15[\mathrm{~m}]$ and $T p=1.4[\mathrm{~s}]$, measured at the device location during preliminary empty-tank simulations

$$
\begin{aligned}
m \ddot{x}(t)=-s_{h} x(t)+ & \mathcal{F}_{\text {exc }}(t)-\mu_{\infty} \ddot{x}(t) \\
& -\int_{-\infty}^{\infty} \zeta(t-\tau) \dot{x}(\tau) d \tau-u(t),
\end{aligned}
$$

where $x(t), \dot{x}(t)$ and $\ddot{x}(t)$ denote the device excursion, velocity and acceleration of the WEC, respectively, $m$ is the mass of the WEC, $s_{h}$ the hydrostatic stiffness, $\mathcal{F}_{\text {exc }}(t)$ the excitation force, $\mu_{\infty}$ the added-mass at infinite frequency, $\zeta(t)$ the radiation impulse response function, and $u(t)$ the control law (PTO force).

For a linear hydrodynamic model, as used herein, all nonlinear effects such as nonlinear Froude Krylov forces or viscous drag are omitted.

\section{B. CNWT}

The CNWT simulations are performed using the open source CFD toolbox OpenFOAM, specifically version 2.3.1 of the OpenFOAM Foundation fork [21]. In OpenFOAM, the incompressible Reynolds Averaged Navier-Stokes (RANS) equations (2) and (3) together with the Volumen-of-Fluid (VOF) method for water wave advection (see Equations (4) and (5)) are solved using the Finite-Volume Method (FVM).

$$
\begin{gathered}
\nabla \cdot \rho \mathbf{U}=0 \\
\frac{\partial(\rho \mathbf{U})}{\partial t}+\nabla \cdot(\rho \mathbf{U} \mathbf{U})=-\nabla p+\nabla \cdot \mathbf{T}+\rho \mathbf{f}_{b} \\
\frac{\partial \alpha}{\partial t}+\nabla \cdot(\mathbf{U} \alpha)+\nabla \cdot\left[\mathbf{U}_{\mathbf{r}} \alpha(1-\alpha)\right]=0 \\
\Phi=\alpha \Phi_{\text {water }}+(1-\alpha) \Phi_{\text {air }}
\end{gathered}
$$

In Equations (2) and (3) $\mathbf{U}$ denotes the fluid velocity, $p$ the fluid pressure, $\rho$ the fluid density, $\mathbf{T}$ the stress tensor, and $\mathbf{f}_{b}$ external forces such as gravity or PTO forces. In the transport Equation (4), $\alpha$ is the water volume fraction and $\mathbf{U}_{\mathbf{r}}$ is the compression velocity [22]. In Equation (5), $\Phi$ is a specific fluid property, such as density. Throughout this study, laminar flow conditions are assumed for all CNWT simulations. The body motion is solved via Newton's 2nd law of motion.

For wave generation and absorption, the relaxation zone method [23], is employed. This method blends a target solution, $\Phi_{\text {target }}$, with the computed solution, $\Phi_{\text {computed }}$, for the values of the velocity field, $\Phi=\mathbf{U}$, and fluid volume fraction, $\Phi=\alpha$, within defined relaxation zone regions (see Fig. 6 a)). A weighting function is zero at the CNWT boundary, unity at the interface with the simulation zone, and should vary smoothly along the relaxation zone to ensure a gradual transition in the blending of the target and computed solutions (see Fig. 6 b)). For wave generation, to define the target solutions, analytical solutions are obtained from wave theories for the fluid velocity and free surface elevation. For wave absorption the target velocity field is zero, and the free surface location is defined at still water level.

Convergence studies were performed to determine the required wave generation $\left(L_{g}\right)$ and wave absorption $\left(L_{a}\right)$ relaxation zone lengths. The spatial and temporal discretisations were similarly determined. The dimensions of the generation and absorption relaxation zone lengths, and the spatial and temporal discretisations, are listed in Table I. The overall spatial dimensions of the CNWT are $7.75[\mathrm{~m}] \mathrm{x}$ $24.92[\mathrm{~m}] \times 6[\mathrm{~m}](\mathrm{WxLxH})$. The numerical domain is depicted in Fig. 6. For further details on the numerical setup and model validation, the interested reader is referred to [17].

TABLE I: Relaxations zone lengths and domain discretisation

\begin{tabular}{lc}
\hline Generation relaxation zone length $\left(L_{g}\right)$ & $3 \lambda$ \\
Absorption relaxation zone length $\left(L_{a}\right)$ & $3 \lambda$ \\
Spatial discretisation: & \\
dx & 200CPLs \\
dy & $50 \mathrm{CPLs}$ \\
$\mathrm{dz}$ & $10 \mathrm{CPHs}$ \\
Temporal discretisation & $1400^{\Delta t} / T$ \\
\hline
\end{tabular}

To realise resistive and reactive controllers in the CNWT, damper respectively spring-damper systems can be employed, which are readily available in OpenFOAM as part of the sixDoFRigidBodyMotionSolver.

Note that in both, the BNWT and CNWT, the control law $u(t)$ directly represents the PTO force. No losses or nonlinear effects in the PTO components are considered. In future work, EMCSs will be evaluated using a high-fidelity simulation platform such as the HiFiWEC, presented in [11]. 
a)

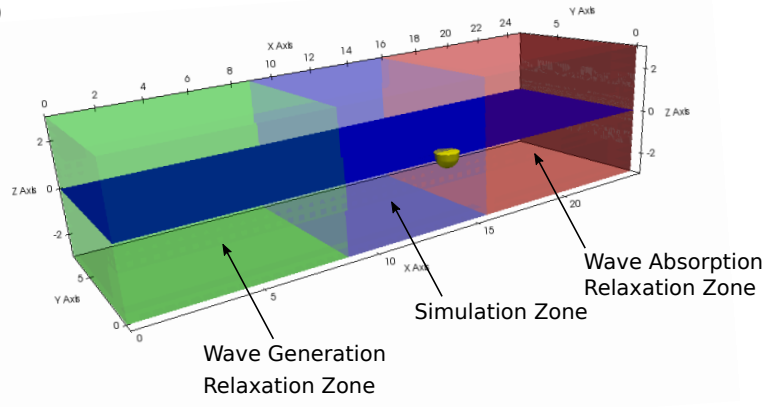

b)

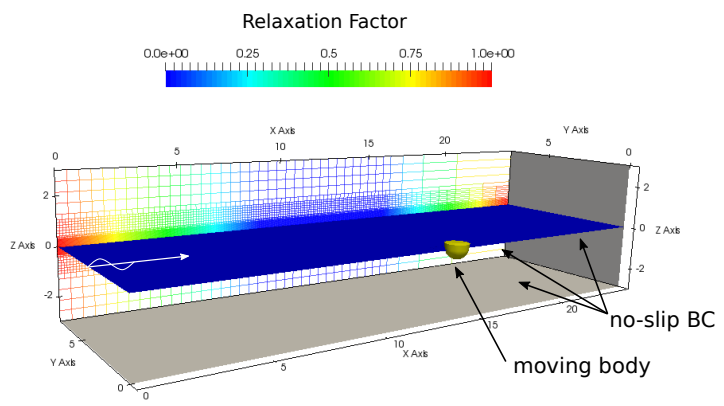

Fig. 6: Visualisation of the CNWT domain including relaxation strengths, still water level and device hull

\section{EMCS}

Throughout the control design, the hydrodynamic model described in Section III-A is considered. The main objective of a wave energy device is to harvest energy from the incoming wave field. ${ }^{1}$ Therefore, the optimal control objective is to maximise the absorbed energy over a time interval $[t, t+T] \subset \mathbb{R}^{+}$, given by

$$
\mathcal{J}=\frac{1}{T} \int_{t}^{t+T} u(\tau) \dot{x}(\tau) d \tau .
$$

while respecting the physical limitations of the device on excursion $x(t)$ and PTO force $u(t)$, i.e. state and input constraints. Note that this energy-maximising control objective does not fit into a traditional (reference tracking) control problem, i.e. the performance objective is strictly related to energy absorption. State and input constraints can be written in compact form as

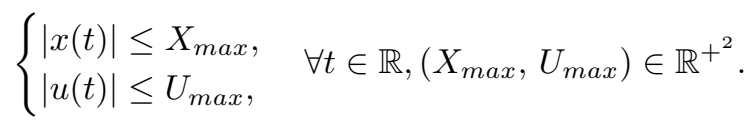

Consequently, the optimal control objective can be formulated as

$$
\begin{aligned}
& u^{\max }(t)=\arg \max _{u(t) \in \mathcal{U}} \mathcal{J} \\
& \text { subject to }\left\{\begin{array}{l}
\text { system dynamics Eqn. (1), } \\
\text { state and input constraints Eqn. (7). }
\end{array}\right.
\end{aligned}
$$

where $\mathcal{U}$ represents the set of admissible inputs. Specifically, for this study, constraints for the stroke length of the hydraulic cylinder $\left(X_{\max }\right)$ and the maximum PTO force $\left(U_{\max }\right)$ are considered during the control design. The PTO cylinder stroke length is set to a maximum of $0.7[\mathrm{~m}]$, while the maximum PTO force is constrained to $2800[\mathrm{~N}]$.

\section{A. Moment-based constrained optimal control}

This constrained optimal control technique relies on the so-called moment-based WEC formulation [14], which allows for an efficient computation of the optimal control law

\footnotetext{
${ }^{1}$ Note, the incoming wave field is measured in an empty CNWT, i.e. no device present, at the device position. It is then assumed that the incoming wave field is not influenced when placing the device in the NWT. This approach bears some inherent errors, however, is common practice.
}

$u^{\max }$ in real-time based on the solution of the following inequality constrained quadratic program:

$$
L_{u}^{\max }=\arg \max _{L_{u}}-\frac{1}{2} L_{u} \Phi_{\varphi}^{\mathscr{R}} L_{u}^{\top}+\frac{1}{2} L_{e x c} \Phi_{\varphi}^{\mathscr{R}} L_{u}^{\top},
$$

subject to:

$$
\begin{aligned}
& L_{u} \Delta \leq U_{\max } \mathbf{1}_{1 \times 2 N_{c}}, \\
& L_{u}\left(-\Phi_{\varphi}^{\mathscr{R}}\right) S^{-1} \Delta \leq X_{\max } \mathbf{1}_{1 \times 2 N_{c}}-L_{e x c} \Phi_{\varphi}^{\mathscr{R}} S^{-1} \Delta .
\end{aligned}
$$

The reader is referred to [14] for the formal definition (and corresponding proofs) of the matrices involved in the QP problem of (9). Note that, differently from [14], where the optimal control input is of a feedforward type, this study uses an equivalent output feedback (displacement and velocity) realisation of the moment-based control input given by $u^{\max }$, which can be readily obtained from $L_{u}^{\max }$ using well-known algebraic identities.

\section{B. Reactive Control}

Additionally to the moment-based controller, less aggressive EMCS, i.e. reactive and resistive controllers, are considered herein as reference cases. For the reactive control case, the PTO force follows

$$
u(t)=K_{\mathrm{opt}} x(t)+B_{\mathrm{opt}} \dot{x}(t),
$$

where $B_{\text {opt }}$ is the optimal damping coefficient and $\dot{x}(t)$ the linear velocity of the hydraulic PTO cylinder, $K_{\mathrm{opt}}$ is the optimal spring stiffness, and $x(t)$ the linear motion of the hydraulic PTO cylinder. The optimal PTO coefficients have been determined through exhaustive search optimisation using the BNWT model, resulting in $K_{\text {opt }}=-33750\left[\mathrm{~N} \mathrm{~m}^{-1}\right]$ and $B_{\text {opt }}=4666\left[\mathrm{~N} \mathrm{~s} \mathrm{~m}^{-1}\right]$. Results of the optimisation are shown in Fig. 7.

\section{Resistive Control}

For the resistive control case, the PTO force follows

$$
u(t)=B_{\mathrm{opt}} \dot{x}(t) .
$$

As in the case of reactive control, the optimal damping coefficient has been determined through exhaustive search optimisation using the BNWT model, resulting in $B_{\text {opt }}=$ $8081\left[\mathrm{~N} \mathrm{~s} \mathrm{~m}^{-1}\right]$. The results of the optimisation are shown in Fig. 8. 


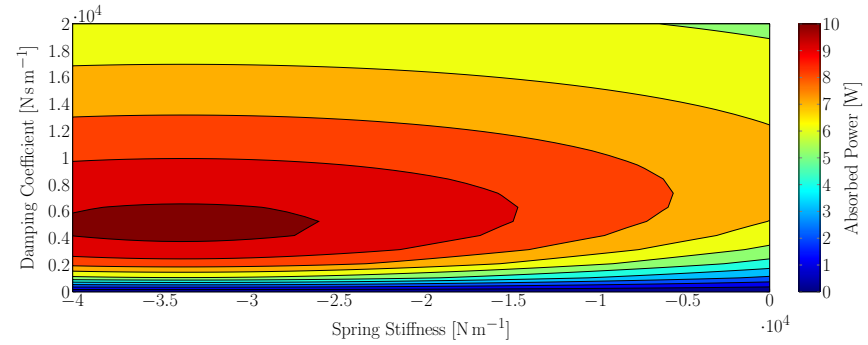

Fig. 7: Contour plot of absorbed power as function of the PTO damping coefficient and spring stiffness for the case of reactive control

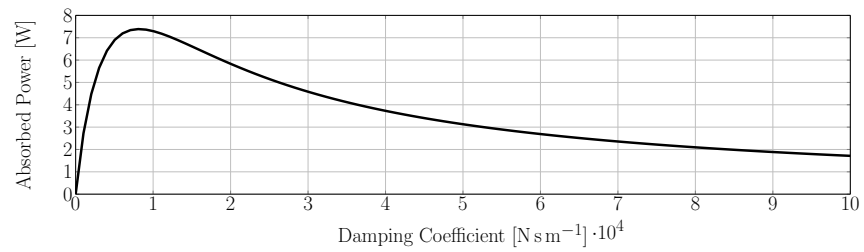

Fig. 8: Absorbed power as function of the PTO damping coefficient for the case of resistive control

\section{RESULTS \& DISCUSSION}

For each EMCS, simulations were performed in both the BNWT and CNWT, resulting in a total of six simulations. Extracting the PTO cylinder displacement, velocity, and the PTO cylinder force, the normalised root mean squared error (nRMSE), as defined in Equation (12), can be calculated. $y_{\mathrm{BNWT}}$ denotes the results from the BNWT experiment, $y_{\text {CNWT }}$ results from the CNWT experiment and $N$ is the length of the signal.

$$
\mathrm{nRMSE}=\frac{\sqrt{\frac{\sum\left(y_{\mathrm{CNWT}}-y_{\mathrm{BNWT}}\right)^{2}}{N}}}{\max \left(y_{\mathrm{BNWT}}\right)-\min \left(y_{\mathrm{BNWT}}\right)}
$$

For qualitative comparison of the three different EMCSs Fig. 9 shows the PTO cylinder displacement, velocity and force extracted from the BNWT experiment. In Fig. 9, a clear trend can be observed. The moment-based and the reactive controller controller increases all three quantities, compared to the resistive controller, driving the WEC furthest away from the assumption of small amplitude motion in the linear hydrodynamic model. The smaller amplitude motion and smallest PTO force can be observed for the case of the resistive controller, since the device effectively acts as a wave follower.

The resulting nRMSE for each PTO quantity for the

TABLE II: nRMSE between the results for PTO cylinder displacement, velocity and PTO force from the BNWT and CNWT for the different EMCSs

\begin{tabular}{llccc}
\hline nRMSE $\left[\cdot 10^{-2}\right]$ & & Moment-based & Reactive & Resistive \\
\hline \hline PTO cylinder displacement & $x$ & 4.53 & 4.50 & 3.20 \\
PTO cylinder velocity & $\dot{x}$ & 3.79 & 3.76 & 2.47 \\
PTO cylinder Force & $u(t)$ & 4.33 & 4.3 & 2.47 \\
\hline
\end{tabular}

different EMCSs are listed in Table II. Overall smallest values of the nRMSE between the BNWT and CNWT results can be observed for the case of a resistively controlled device. For the PTO cylinder displacement, velocity and force the nRMSEs are, respectively, $3.2 \cdot 10^{-2}, 2.47 \cdot 10^{-2}$ and $2.47 \cdot 10^{-2}$. For this control strategy, smallest errors are indeed expected, since the device effectively acts as a wave follower, reducing the influence of non-linear hydrodynamic effects, such such as non-linear Froude-Krylov forces or viscous drag effects.

For the cases with reactive and moment-based control, the values for the nRMSE in all PTO quantities increase. For the PTO cylinder displacement, the nRMSEs are, respectively, $4.50 \cdot 10^{-2}$ and $4.53 \cdot 10^{-2}$, for the reactive and momentbased controller. For the PTO cylinder velocity, the nRMSEs are $3.76 \cdot 10^{-2}$ for the reactive controller and $3.76 \cdot 10^{-2}$ for the moment-based controller. For the PTO cylinder force, the nRMSEs are $4.50 \cdot 10^{-2}$ and $4.53 \cdot 10^{-2}$, for the reactive and moment-based controller, respectively. From the time traces shown in Fig. 9, it can be seen that the differences between the PTO quantities for the two different controllers are negligible. This can be attributed to the fact that both state (displacement) and input (PTO force) constraints are inactive for this particular input wave, i.e. both controllers effectively reflect the unconstrained optimal energy-absorption conditions. That said, it is expected that also the differences between the BNWT and CNWT are similar.

Furthermore, the larger errors for the cases with reactive and moment-based control, compared to the case with resistive control, underlining that more aggressive control strategies lead to larger deviations between BNWT and CNWT experiments. The larger deviations can be reasoned with the increased WEC motion amplitude and PTO forces (see Fig. 9), driving the system further away from the assumptions in the linear hydrodynamic model, resulting in a larger mismatch between BNWT and CNWT results.

\section{CONCLUSIONS}

This paper studied the influence of two different numerical frameworks, i.e. a linear BNWT and a CNWT, on the evaluation of three different EMCSs. Discrepancies in the performance, by means of PTO cylinder displacement, velocity and force, were observed when using different modelling environments for the evaluation. A correlation between aggressiveness of the EMCS and the deviations between linear BNWT and CNWT can be seen. More aggressive control strategies lead to increased device motion, driving the WEC further away from the assumption of the linear hydrodynamic model and thus show larger deviations between BNWT and CNWT experiments. It can be concluded, that the choice of the numerical modelling framework for both design and evaluation of EMCSs has a significant influence on the performance of the controller and should be chosen carefully.

\section{ACKNOWLEDGMENT}

This paper is based upon work supported by Science Foundation Ireland under Grant No. 13/IA/1886. 

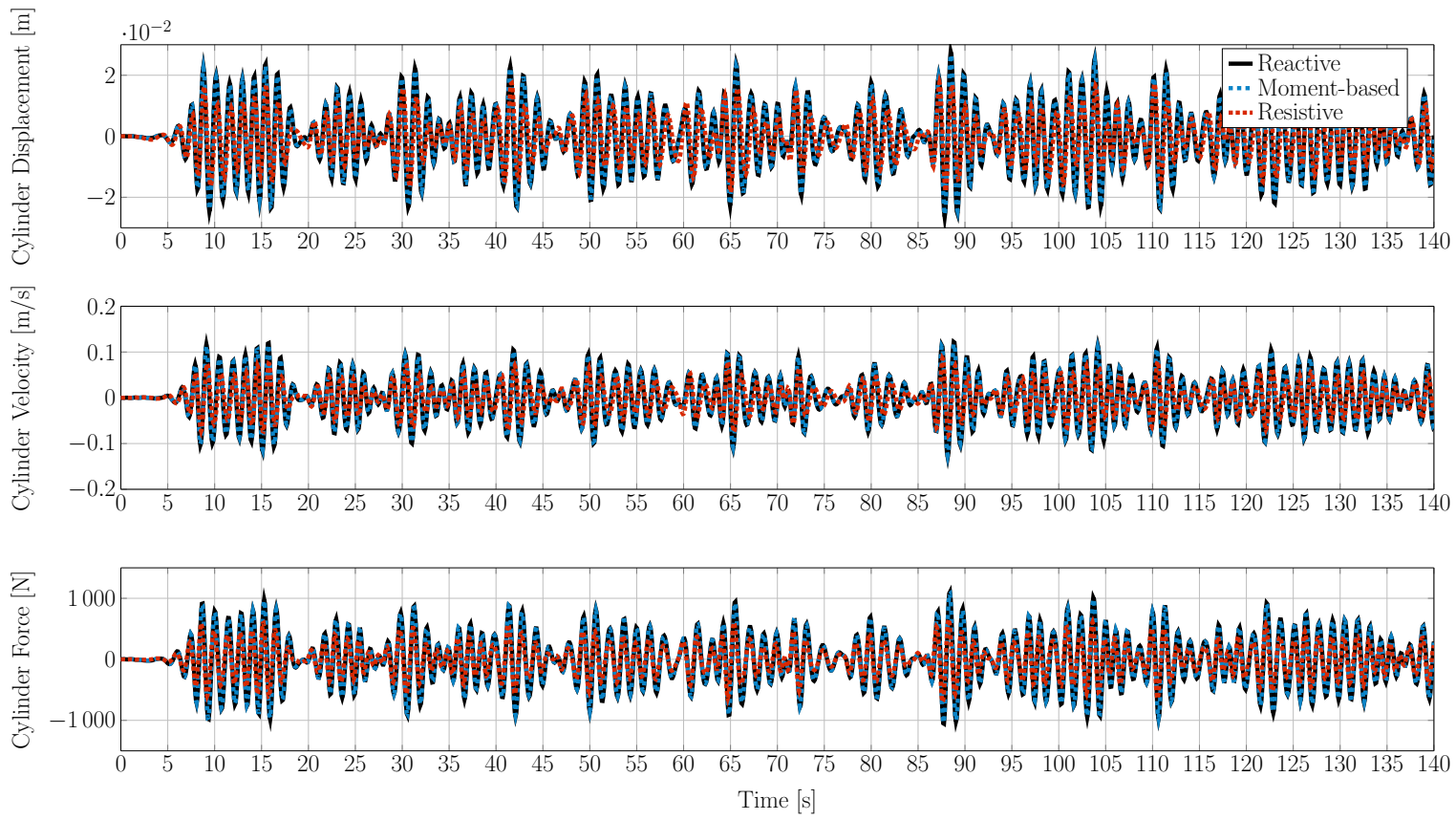

Fig. 9: Time traces of the PTO cylinder displacement $x(t)$, velocity $\dot{x}(t)$, and the PTO cylinder force $u(t)$ from the BNWT experiment

\section{REFERENCES}

[1] A. F. de O. Falcão, "Wave energy utilization: A review of the technologies," Renewable and Sustainable Energy Reviews, vol. 14, pp. 899-918, 2010.

[2] K. Ruehl and D. Bull, "Wave Energy Development Roadmap: Design to commercialization," OCEANS 2012 MTS/IEEE: Harnessing the Power of the Ocean, 2012.

[3] J. V. Ringwood, G. Bacelli, and F. Fusco, "Energy-maximizing control of wave-energy converters: The development of control system technology to optimize their operation," IEEE Control Systems, vol. 34, pp. 30-55, 2014.

[4] G. Giorgi and J. V. Ringwood, "Implementation of latching control in a numerical wave tank with regular waves," Journal of Ocean Engineering and Marine Energy, vol. 2, pp. 211-226, 2016.

[5] _ - "Computationally efficient nonlinear Froude-Krylov force calculations for heaving axisymmetric wave energy point absorbers," Journal of Ocean Engineering and Marine Energy, vol. 3, no. 1, pp. 21-33, February 2017.

[6] J. R. Morison, M. P. O'Brien, J. W. Johnson, and S. A. Schaaf, "The forces exerted by surface waves on pliles," Petroleum Trans., AIME. Vol. 189, pp. 149-157, 1950.

[7] J. Davidson, C. Windt, G. Giorgi, R. Genest, and J. Ringwood, "Evaluation of energy maximising control systems for wave energy converters using OpenFOAM," in Selected papers of the 11th OpenFOAM Workshop. Springer International Publishing AG, 2017.

[8] J. Davidson, R. Genest, and J. Ringwood, "Adaptive control of a wave energy converter simulated in a numerical wave tank," in Proceedings of the 12th European Wave and Tidal Energy Conference, Cork, 2017, pp. 747-1 - 747-10.

[9] I. Dolguntseva, W. Chen, A. Savin, Y. Zhang, W. Li, O. Svensson, and M. Leijon, "Numerical modelling of a point-absorbing wave energy converter in irregular and extreme waves," Applied Ocean Research, vol. 63, pp. 90 - 105, 2017.

[10] C. Eskilsson, W. Wang, M. Wu, and J. Palm, "Estimation of numerical uncertainty in CFD simulations of a passivley controlled wave energy converter," Proceedings of the Institution of Mechanical Engineers, Part M: Journal of Engineering for the Maritime Environment, vol. 232, pp. 71-84, 2017.

[11] M. Penalba, J. Davidson, C. Windt, and J. Ringwood, "A highfidelity wave-to-wire simulations platform for wave energy converters: Coupled numerical wave tank and power take-off models," Applied Energy, vol. 226, pp. 655-669, 2018.
[12] C. Windt, J. Davidson, D. Chandar, and J. Ringwood, "On the importance of advanced mesh motion method for wec experiments in CFD-based numerical wave tanks," Submitted to the VIII International Conference on Computational Methods in Marine Engineering, Gothenburg, 2019.

[13] N. Faedo, S. Olaya, and J. V. Ringwood, "Optimal control, MPC and MPC-like algorithms for wave energy systems: An overview," IFAC Journal of Systems and Control, vol. 1, pp. 37-56, 2017.

[14] N. Faedo, G. Scarciotti, A. Astolfi, and J. V. Ringwood, "Energymaximising control of wave energy converters using a moment-domain representation," Control Engineering Practice, vol. 81, pp. 85-96, 2018.

[15] J. Hals Todalshaug, A. Babarit, J. Krokstad, M. J. Muliawan, A. Kurniawan, and T. Moan, "The NumWEC project. Numerical estimation of energy delivery from a selection of wave energy converters," Norwegian University of Science and Technology, Tech. Rep., 2015.

[16] M. M. Jakobsen, S. Beatty, G. Iglesias, and M. M. Kramer, "Characterization of loads on a hemispherical point absorber wave energy converter," International Journal of Marine Energy, vol. 13, pp. 1-15, 2016.

[17] C. Windt, J. Davidson, E. Ransley, D. Greaves, M. Jakobsen, M. Kramer, and J. Ringwood, "Validation of a CFD-based numerical wave tank model of the Wavestar ocean wave energy converter," submitted to Renewable Energy, 2018.

[18] M. M. Jakobsen, "Wave-Structure interations on point absorber," Aalbor University Denmark, Tech. Rep., 2014.

[19] J. Falnes, Ocean Waves and Oscillating Systems: Linear Interactions Including Wave-Energy Extraction. Cambridge University Press, 2002.

[20] W. E. Cummins, "The impulse response function and ship motions," DTIC Document, Tech. Rep., 1962.

[21] The OpenFOAM Foundation, "OpenFOAM foundation webpage," 2018, last accessed 08.02. [Online]. Available: https://openfoam.org/

[22] E. Berberović, N. P. van Hinsberg, S. Jakirlić, I. V. Roisman, and C. Tropea, "Drop impact onto a liquid layer of finite thickness: Dynamics of the cavity evolution," Physical Review E, vol. 79, pp. 036306-1 - 036306-15, 2009.

[23] N. Jacobsen, D. R. Fuhrmann, and J. Fredsoe, "A wave generation toolbox for the open-source CFD library: OpenFoam(R)," International Journal for Numerical Methods in Fluids, vol. 70, pp. 1073-1088, 2012. 